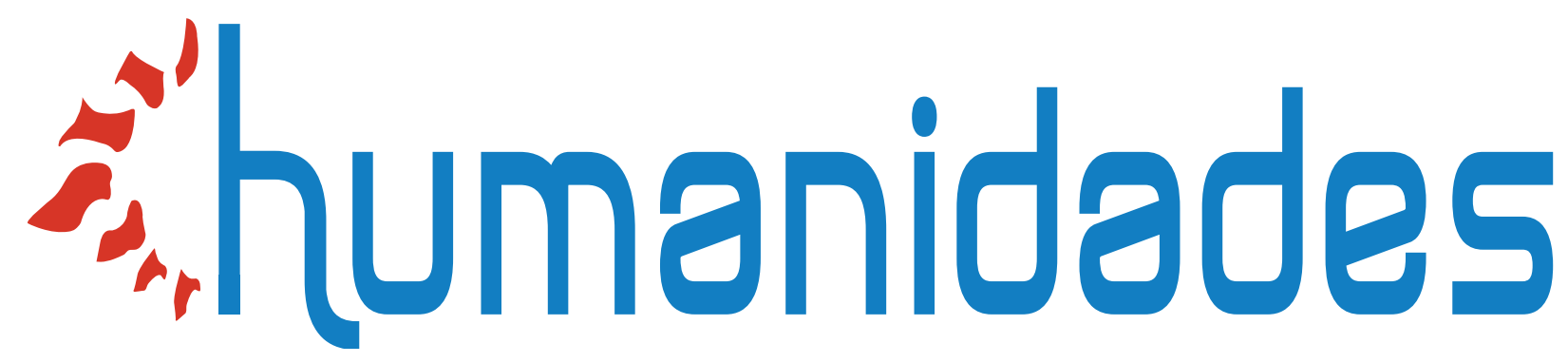

\title{
Apreciaciones del profesorado chileno sobre una educación basada en el amor: estudio de casos
}

Felipe Mujica Johnson

Nelly Orellana Arduiz

DOI: https://doi.org/I0.155 I7/h.v10il.392I5 https://revistas.ucr.ac.cr/index.php/humanidades/index 


\section{"humanidades}

Revista Humanidades

ISSN: 2215-3934

humanidades@ucr.ac.cr

Universidad de Costa Rica

Costa Rica

\section{Apreciaciones del profesorado chileno sobre una educación basada en el amor: estudio de casos}

Mujica Johnson, Mag. Felipe; Orellana Arduiz, Dra. Nelly

Apreciaciones del profesorado chileno sobre una educación basada en el amor: estudio de casos

Revista Humanidades, vol. 10, núm. 1, 2020

Universidad de Costa Rica, Costa Rica

Disponible en: http://www.redalyc.org/articulo.oa?id=498060395005

DOI: https://doi.org/10.15517/h.v10i1.39215

Todos los derechos reservados. Universidad de Costa Rica. Esta revista se encuentra licenciada con Creative Commons. Reconocimiento-NoComercial-SinObraDerivada 3.0 Costa Rica. Correo electrónico: humanidades@ucr.ac.cr/Sitio web: http: //revistas.ucr.ac.cr/index.php/humanidades

Esta obra está bajo una Licencia Creative Commons Atribución-NoComercial-SinDerivar 3.0 Internacional. 


\title{
Apreciaciones del profesorado chileno sobre una educación basada en el amor: estudio de casos
}

\author{
Appreciations of Chilean Teachers on an Education Based on Love: Case Studies
}

Mag. Felipe Mujica Johnson

Universidad Politécnica de Madrid, España

felipe.mujica@upla.cl

(iD http://orcid.org/0000-0002-6956-2357

Dra. Nelly Orellana Arduiz

Universidad de Playa Ancha de Ciencias de la Educación,

Chile

norellan@upla.cl

(iD) http://orcid.org/0000-0002-5742-4539
DOI: https://doi.org/10.15517/h.v10i1.39215

Redalyc: http://www.redalyc.org/articulo.oa? id $=498060395005$

Recepción: 28 Mayo 2019

Aprobación: 03 Septiembre 2019

\section{Resumen:}

El amor como parte de la dimensión afectiva del ser humano requiere ser incorporado significativamente en la labor pedagógica, no obstante, es un desafío complejo por la naturaleza de aquel afecto, por lo que este estudio pretende comprender la perspectiva del profesorado. El propósito del estudio es identificar la representación social del amor en la educación escolar por parte del profesorado que se desempeña en la educación pública. El estudio responde al enfoque cualitativo y sus participantes son seis docentes de diferentes disciplinas pedagógicas. La recolección de datos se realizó por medio de informes cualitativos y entrevistas semi-estructuradas. Los datos fueron sometidos a un análisis de contenido que constó de tres fases utilizando el programa Atlas.ti 7.5. Los resultados presentan dos familias, semántica del amor y rol del amor en la educación escolar, las cuales se encuentran compuestas por tres códigos cada una. Se concluye que los docentes perciben el amor como un componente esencial para el bienestar y el aprendizaje, el cual es construido socialmente y se transmite por medio del lenguaje, transformando la realidad educativa.

Palabras ClaVe: Afectividad, Educación Formal, Bienestar del estudiante, Docente, educación moral.

\section{Abstract:}

Love as part of the affective dimension of the human being needs to be incorporated significantly into the pedagogical work, however, it is a complex challenge because of the nature of that affect, so this study aims to understand the perspective of the teaching staff. The purpose of the study is to identify the social representation of love in school education by teachers who work in public education. The study responds to the qualitative approach and its participants are six teachers from different pedagogical disciplines. The data collection was done through qualitative reports and semi-structured interviews. The data were subjected to a content analysis consisting of three phases, using the Atlas.ti 7.5 program. The results present two families, semantics of love and the role of love in school education, which are composed of three codes each. It is concluded that teachers perceive love as an essential component for wellbeing and learning, which is socially constructed and transmitted through language, transforming the educational reality.

KEYWORDS: Emotions, Formal education, Student welfare, Teachers, Moral Education.

\section{INTRODUCCIÓN}

La afectividad no es un tema que haya sido priorizado en la historia de la educación chilena, sin embargo, en las últimas décadas con el reconocimiento de la interacción que existe entre razón y emoción (Maturana, 2001; Mora, 2017; Mujica, 2018), se ha trabajado por reconocer su importancia en el quehacer educativo. 
Respecto a su significado, es definida como "un conjunto de emociones, estados de ánimo y sentimientos que permean los actos de las personas, incidiendo en el pensamiento, la conducta y la forma de relacionarse con uno mismo y con los demás" (Ministerio de Educación, 2013). Esta dimensión se encuentra presente en el marco legal que establece las finalidades de la educación en el país, pudiendo apreciarse en la ley general de educación $n^{\circ} 20.370$, promulgada el 16 de diciembre del año 2009. En concreto, esta ley establece que la comunidad escolar debe tener un objetivo común, que es "contribuir a la formación y el logro de aprendizajes de todos los alumnos que son miembros de esta, propendiendo a asegurar su pleno desarrollo espiritual, ético, moral, afectivo, intelectual, artístico y físico" (Ministerio de Educación, 2010, p. 5). En otros términos, señala que es fundamental lograr la educación integral de las personas, respetando la importancia que tienen los diferentes ámbitos que integran la existencia del ser humano (Mujica, 2018). Por lo tanto, no menospreciando la dimensión afectiva y corporal, al modo del racionalista dualismo cartesiano (Descartes, 2011). Este dualismo al que se hace mención ha tenido una notable influencia en el pensamiento occidental, atribuyéndosele la poca consideración que tiene la afectividad en los procesos educativos, aunque se ha ido desplazando paulatinamente conlas aportaciones realizadas por la filosofía y las ciencias cognitivas (Scheler, 2005). Precisamente, estos aportes han generado conocimiento que describe cómo el sistema emocional interviene en la deliberación tradicionalmente entendida como exclusivamente racional (Cabezas, 2013). Sobre este asunto, se refiere Ferrés y Masanet (2017), agregando que los descubrimientos más sorprendentes de la neurociencia manifiestan la importancia que tienen las emociones y el inconsciente en los procesos mentales, incluidos los procesos racionales. Entonces, estas ideas desafían a re-pensar el proceso educativo, reflexionando sobre los aspectos teóricos que guían las acciones pedagógicas, así como también incorporando la mirada de los actores que se recoge a través de las investigaciones educativas.

Para comprender desde una perspectiva cultural el desarrollo histórico en que se encuentra la sociedad con relación al entendimiento de las emociones y el mundo afectivo, Zaccagnini (2008) ha realizado una breve síntesis histórica del tema en la sociedad occidental. Esta se resume en los siguientes puntos: a) Grecia Clásica, las emociones eran entendidas como pasiones incontrolables que debían de ser aceptadas; b) Edad Media, las emociones fueron asociadas a impulsos negativos o tentaciones asociadas al pecado, de modo que debían ser vencidas; c) Modernidad, las emociones eran impulsos irracionales que atentaban contra la razón, de modo que había que reprimirlas; y d) Posmodernidad, las emociones interactúan con la razón y el medio ambiente, de modo que es preciso comprenderlas y controlarlas para optimizar la calidad de vida.

Ahora bien, cabe destacar, que a pesar de que en la modernidad prosperó la perspectiva cartesiana, hubo filósofos como Pascal (1967) que siguieron una línea diferente de argumentación, y sostuvieron la relevancia que tiene la afectividad en la vida humana, así como también su vínculo con la trascendencia del alma. Siguiendo esta argumentación sobre la importancia de la afectividad, destaca Scheler (2005), (filósofo alemán) que en el siglo XX defiende una ética objetiva y material, postulando diferentes categorías jerarquizadas de los sentimientos humanos. Entre esas categorías, el amor sería parte de los sentimientos espirituales, de modo que sería un sentimiento objetivo y por lo tanto independiente de la conciencia humana. Asimismo, la categoría espiritual del amor estaría sobre los sentimientos psicológicos y subjetivos, como las emociones, de esta forma, el amor tendría la capacidad de suscitar el buen desarrollo moral de las personas y por consiguiente, buenas emociones (Mujica, 2019). Por lo tanto, desde esta perspectiva scheleriana el amor sería entendido de la siguiente forma:

El amor es un proceso intencional que va más allá de lo que se ama; lo que se ama es valorado positivamente; lo mismo que el odio es algo que nace, pero de un valor negativo, desvalorado. Es un acto o un conjunto de actos de la voluntad que se inclina intencionadamente hacia él. (Rojas, 1993, p. 87)

En cuanto a la comprensión de las emociones que ha construido la posmodernidad, se encontraría en parte reflejada en la corriente pedagógica de la "educación emocional” (Bisquerra, 2005). Esta corriente postula que el ser humano debe desarrollar capacidades y competencias en torno a sus vivencias emocionales, incluyendo 
también la formación del profesorado, que tendría que concluir en un profesional competente en el tema y, además, que sepa desarrollar esas competencias en sus aprendices. Las competencias emocionales han sido clasificadas en cinco sub-dimensiones por Bisquerra y Pérez (2007) que son: la conciencia emocional; la regulación emocional; la autonomía emocional; la competencia social; y las competencias para la vida y el bienestar.

Con respecto al significado del amor, si se entiende "como una emoción que constituye las acciones de aceptar al otro como un legítimo otro en la convivencia" (Maturana, 2001, p. 46), entonces, es posible considerar que la competencia más vinculada a un docente que practica una educación basada en el amor, es la competencia social. Esta última es entendida como "la capacidad para mantener buenas relaciones con otras personas. Esto implica dominar las habilidades sociales, capacidad para la comunicación efectiva, respeto, actitudes pro-sociales, asertividad, etc." (Bisquerra y Pérez, 2007, p. 72). Este asunto de la competencia emocional cobra sentido al reconocer las difíciles condiciones en las que educan los docentes en la actualidad, marcadas por la inasistencia del alumnado, la falta de motivación por el estudio, inadecuadas relaciones personales, ambientes desfavorables para el aprendizaje y problemas psicosociales del alumnado (Hernández, 2017).

Una relación del amor con el rol docente en la educación escolar en Chile, según declara el Ministerio de Educación (2009) con relación al ámbito de crecimiento y autoafirmación personal, sería "profundizar en el sentido y valor del amor y de la amistad" (Ministerio de Educación, 2009, p. 23). El problema con relación a lo anterior se genera en el significado que cada docente le atribuya al amor, ya que no se presenta una aclaración semántica de lo que significa el amor de forma general. Justamente, el significado personal que tenga el profesorado con respecto al tema estará asociado a su representación social, construida a lo largo de su vida, la cuale corresponde a "una forma específica de conocimiento, el conocimiento ordinario, que es incluido en la categoría del sentido común y tiene como particularidad la de ser socialmente construido y compartido en el seno de diferentes grupos" (Jodelet, 2011, p. 134). Entre los factores que contribuyen a las representaciones se encuentran las relaciones sociales, con mayor énfasis en las que se producen con el grupo de amigos y amigas, los padres y madres, la escuela, las organizaciones deportivas o culturales, los grupos religiosos o filosóficos, las iglesias y los medios de comunicación (Vergara, 2008). Debido a la necesidad de visibilizar aquellos significados, para discutirlos a nivel conceptual y encontrar sentidos que permitan su transferencia en la práctica, es que se abordarán con un enfoque cualitativo, entendiendo que cada interpretación del tema ayudará a su comprensión.

\subsection{El amor en educación}

El concepto del amor ha sido utilizado en nuestra larga historia con múltiples significados, adecuándose a los diferentes contextos, de esta manera, resulta lógico entenderlo como un "concepto diverso, repleto de contrastes, antítesis, paradojas y peculiaridades, por lo que resulta tan singular y complejo a la vez" (Chalita, 2006, p. 13). Una concepción del amor que puede ser considerada en la lógica educativa por el hecho de que aspira a un desarrollo personal, que trascienda la conducta egoísta, para alcanzar un comportamiento solidario y pro-social, es el amor fraternal, que se encuentra fundado en "el sentido de responsabilidad, cuidado, respeto y conocimiento con respecto a cualquier otro ser humano, el deseo de promover su vida" (Fromm, 1959, p. 52). Los valores mencionados anteriormente son universalmente conocidos e incluidos en los espacios educativos, por lo que vincularlos al plano afectivo sería de gran utilidad para que se reconozca el trasfondo emocional que los impulsa. Más aun, cuando estos sentimientos se encuentran integrados a los razonamientos que ayudan a lograr un desarrollo moral del alumnado (Maturana, 2001; Mujica, 2019; Scheler, 2005). Uno de los errores que impiden vincular los valores a la emoción en la educación escolar, es creer que los juicios morales "incorrectos" en algún sentido son fruto de la emoción y los "correctos" de la razón, lo que debe ser modificado pues, razón y emoción sustentan cualquier juicio 
moral (Cabezas, 2013). Si el cuerpo docente ejerce su profesión solo desde un enfoque de capacitación de habilidades académicas y se olvida de la formación en valores de la persona, la necesidad de que haya una práctica pedagógica basada en el amor desaparece, ya que un enfoque fundamentado en la obediencia sería suficiente, debido a que según Maturana (2001):

Los valores de la vida cotidiana se fundan en el amor. El respeto se da en la aceptación del otro como un legítimo otro en la convivencia, y donde hay colaboración que se da sólo en el respeto mutuo, desaparecen la arrogancia y la obediencia. (p. 264).

Una propuesta docente más reciente que establece un acercamiento al niño desde el amor con corazón, es la que plantea Toro (2017), invitando a recuperar el componente emotivo de la racionalidad, por medio de una integración crítica (razón-emoción; cuerpo-mente). Así, se haría frente a una tendencia al reduccionismo, puesto que el sentir más íntimo y profundo, se encuentra ignorado, relegado, desvalorizado, ausente de lo que se vive cotidianamente en muchas escuelas e instituciones educativas. La relación entre una educación basada en el amor y los valores humanos se ha presentado como un aspecto que no solo pretende que el aprendizaje sea más eficaz, sino que optimiza la calidad educativa en la vertiente cultural. Por ejemplo, generar una integración con las personas que emigran a una cultura diferente, asunto que cada vez es más común. Educar en el amor desde esta perspectiva, significaría que el profesorado desarrolle un conjunto de acciones basadas en la ética, el afecto, el respeto, la comprensión y la ayuda hacia el alumnado de manera altruista, fundamentada en una vocación y amor por su profesión (Araque, 2010; Mujica y Orellana, 2019).

Un fundamento esencial para considerar el amor en la educación, es la naturaleza amorosa del ser humano, ya que gracias a esta emoción básica hemos sobrevivido como especie. Esta emoción nos expande y nos conecta a otras personas, experimentando una sensación de unión, la cual no solo es fuente de apoyo social, sino que incrementa el bienestar y protege a las personas del estrés y de las enfermedades (Carpena, 2016, p. 80). Otro de los planteamientos que respaldan la relación amor-educación y por ende la inclusión de esta primera en las prácticas pedagógicas es la de Ortiz (2009), quien señala que el amor es una competencia humana. Una competencia pedagógica que debe ser desarrollada por todo maestro y padre de familia. Esta discusión que tiene como base la educación emocional y la inclusión de las emociones en la pedagogía va en aumento, de modo que requiere ser trasladada adecuadamente a las instituciones educativas. Sobre todo valorando las apreciaciones docentes, porque el pensamiento del profesorado que se desempeña a diario en la tarea de educar, tiene mucho valor y significancia. De esta forma se podrá comprender y establecer mejoras de calidad al sistema educativo chileno y de otros países interesados en esta problemática.

A partir del marco conceptual presentado se puede apreciar que es necesario indagar en la interpretación del amor en la educación formal, razón por la que a través de este estudio se pretende comprender los significados en torno al amor en la educación escolar. En concreto, por parte de un grupo de profesorado chileno que desempeña su labor en educación pública. De esta forma, se podrán identificar dimensiones semánticas sobre el constructo del amor aplicado a la educación, su vinculación con el quehacer didáctico y las vivencias en torno al tema desde el rol docente. Así, este conocimiento representará tanto el pensar como el sentir de personas que cumplen un rol muy complejo y relevante en el sistema educativo escolar.

\section{Materiales y método}

La investigación responde al enfoque cualitativo, ya que es necesario indagar en las concepciones subjetivas no directamente cuantificables que cada docente presenta sobre la función del amor en la educación. Y este tipo de metodología "nos permite una comprensión intersubjetiva que emerge a partir de toda la información analizada, entendiendo que la misma esconde un contenido caracterizado implícita o explícitamente, por las intencionalidades educativas, finalidades, concepciones, exclusiones, valoraciones, etc." (Moreno y Poblete, 2015, p. 292). El paradigma en que se adscribe el estudio es en el de la fenomenología interpretativa, la cual es entendida como una episteme de las ciencias sociales y humanas, que tiene como principal objeto "la 
conciencia de todas las formas de vivencias, actos y correlatos de los mismos, es una ciencia de esencias que pretende llegar sólo a conocimientos esenciales y no fijar, en absoluto, hechos" (Barbera y Inciarte, 2012, p. 204). La perspectiva teórica seleccionada es la del estudio de caso (Mujica y Orellana, 2019), específicamente a nivel colectivo, ya que este enfoque pretende lograr el estudio del amor desde la particular y compleja perspectiva docente, para llegar a comprender su actividad en circunstancias importantes, realizando una interpretación colectiva del tema (Stake, 1995; Stake, 2007).

\subsection{Participantes}

Los participantes del estudio fueron seleccionados en forma intencionada, dada la naturaleza de la investigación (Jordán y Méndez, 2017), por su buen desempeño en la educación escolar del sector público. Estos fueron reconocidos por terceras personas que han desempeñado labores educativas junto a ellos como profesionales comprometidos con la educación pública. Además, son de confianza de los investigadores, como profesionales que comprenden y construyen con su quehacer cotidiano una educación desde una concepción holística (educación no solo para aprender contenidos técnicos o disciplinares, sino que una educación para la vida), donde la relación alumnado-profesorado es muy relevante para los logros escolares. Igualmente, para la selección de los participantes se consideró su especialidad pedagógica para adoptar las percepciones desde diferentes roles docentes. Se establecieron estos criterios de selección, para asegurar que el profesorado participante fuese sensible al fenómeno estudiado y pudiese implicarse con significados relevantes al tema, aportando sus más profundas reflexiones, las cuales, a su vez, surgen de sus experiencias personales, de las que siempre queda una apreciación personal que interpreta en forma crítica lo que está sucediendo en el aula.

Los docentes que participaron son un total de seis, distribuidos de la siguiente manera: un profesor del Departamento de Educación Física con 16 años de experiencia en el sistema educativo escolar de la ciudad de Puerto Montt; un profesor del Departamento de Educación Musical con 4 años en el sistema educativo escolar de la ciudad de Valparaíso; un profesor del Departamento de Educación Artística con 35 años de experiencia en el sistema escolar de la ciudad de Valparaíso; una Profesora del Departamento de Educación General Básica con 30 años de experiencia en el sistema educativo escolar de la ciudad de Viña del Mar; una Psicopedagoga que se encuentra autorizada por el Ministerio de Educación para desempeñarse como docente en el Departamento de Educación Diferencial, la cual cuenta con 10 años de experiencia en el sistema educativo escolar de la ciudad de Viña del Mar; y por último, una Profesora del Departamento de Educación Parvularia con 30 años de experiencia en el sistema educativo escolar de la ciudad de Viña del Mar. Todo el profesorado informante desempeña su labor en establecimientos municipales (públicos) de Chile, los cuales tienen un total financiamiento estatal. Para las consideraciones éticas del estudio los participantes firmaron un consentimiento informado en donde toman conocimiento de los fines investigativos del proceso, de la confidencialidad de los datos y proceden a aceptar voluntariamente la participación en el estudio.

\subsection{Procedimientos y técnicas de recolección de datos}

Para el trabajo de campo se utilizó como primera técnica de recolección de datos un informe cualitativo o no-estructurado ad-hoc, en el cual se plantearon preguntas abiertas y le permitió al sujeto la posibilidad de emitir la respuesta en sus propios términos, pero recordándoles que estas deben de ser de la mayor claridad y precisión posibles (Moreno, 2000). La segunda técnica de recolección de datos, fue la entrevista semiestructurada. Para la construcción de las preguntas del informe y la entrevista, se emplearon dos dimensiones: a) semántica del amor en la educación escolar, que se refiere a los significados que le otorgan los docentes según sus concepciones personales al constructo estudiado; b) rol del amor en la educación escolar, en la que se contempla su funcionalidad en el quehacer educativo. Las preguntas estuvieron orientadas a obtener 
información relevante para las dimensiones de la investigación. La construcción de las categorías se realizó a medida que se recogieron los datos, debido a que el proceso de codificación responde a la vía inductiva (Anguera, Arnau, Ato, Martínez, Pascual y Vallejo, 1998). Para la aplicación de las entrevistas se consideraron las recomendaciones que realiza Vargas-Jiménez (2012), de generar un ambiente agradable que se encuentre enmarcado en el respeto, propiciando la seguridad y confianza al entrevistado. Además, utilizando un guión flexible, manteniendo una escucha activa, ya que de lo contrario se estaría dificultando la comunicación. Para el registro del audio se utilizó la grabadora de voz digital Sony ICD-PX240//CLA.

\subsection{Análisis de datos}

Se llevó a cabo un análisis de contenido que tuvo tres fases, la primera consiste en identificar temas, desarrollando conceptos y proposiciones. La segunda fase se produce una vez ya recogidos lo datos, la cual consiste en codificar los datos y el refinamiento de la comprensión del tema de estudio. Y como última fase el investigador intenta comprender los datos en el contexto en el que fueron recogidos. En la investigación cualitativa, la codificación es un modo sistemático de desarrollar y refinar las interpretaciones de los datos (Bogdan y Taylor, 2009).

Para optimizar la codificación, relación y teorización de los datos, se utilizó el programa informático Atlas.ti versión 7.5, el cual es reconocido por su alcance en el análisis cualitativo (Pérez-López, MoralesSánchez, Anguera y Hernández-Mendo, 2016). Este análisis tuvo como punto de partida la lectura y la transcripción de los informes cualitativos, luego, se continuó con la escucha de los audios de las entrevistas y su posterior transcripción. A medida que se revisó la información se comenzaron a elaborar relaciones e interpretaciones de los relatos, para lo cual se generó un registro de notas que se emplearon en las fases posteriores de análisis. De esta forma, se evitó cometer el error de comenzar en un tiempo tardío el análisis (Rapley, 2014). Las transcripciones del discurso docente establecieron los documentos primarios y que al ser ingresados al programa de análisis Atlas.ti, dichos elementos generaron la base de la unidad hermenéutica que se presenta en la Figura 1. La calidad del estudio en la codificación fue controlada desde la perspectiva cualitativa con la fiabilidad inter-observadores, a través de la concordancia consensuada. En esta estrategia coinciden diferentes codificadores a la hora de analizar el material, estando presentes en el mismo lugar y tiempo (García-Fariña, Jiménez y Anguera, 2016). Para este estudio, dos observadores codificaron conjuntamente un $50 \%$ del discurso del profesorado. 


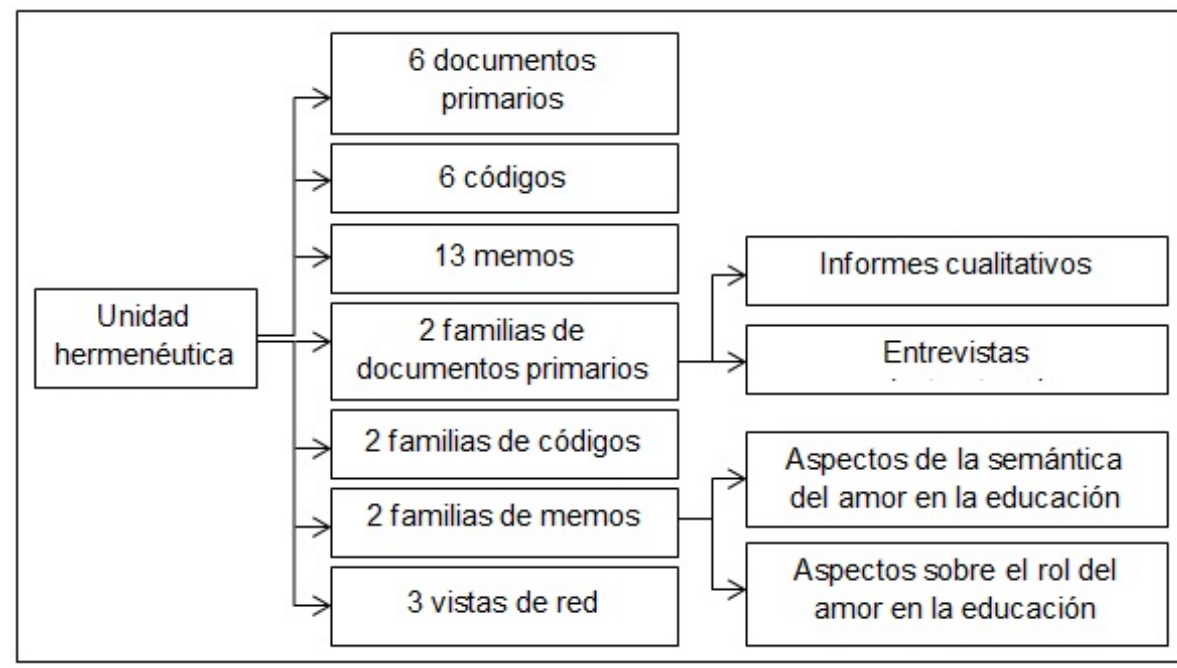

FIGURA 1

Unidad hermeéutica del análisis de contenido

Fuente: Elaboración propia

\section{Resultados}

El proceso de codificación de los datos generó la construcción de seis códigos, los cuales fueron agrupados en dos familias que representan las dimensiones del estudio. Estas son a) semántica del amor en la educación escolar; b) rol del amor en la educación escolar. A continuación se presenta una descripción de los resultados en la Tabla 1, que incluye los códigos y citas obtenidas con el programa Atlas.ti.

TABLA 1

Codificación de las representaciones del amor en la educación escolar.

\begin{tabular}{lll}
\hline Familias & Códigos & Citas \\
\hline Semántica del amor en la & Sentimiento de bienestar subjetivo & 17 \\
educación escolar & Sentimiento transmisible & 14 \\
& Construcción en la interacción social & 19 \\
\hline Rol del amor en la & Vocacional & 20 \\
educación escolar & Favorece el aprendizaje & 18 \\
& Rol irrelevante para la institucionalidad & 14 \\
\hline
\end{tabular}

Fuente: Elaboración propia

\subsection{Semántica del amor en la educación escolar}

Los significados que el profesorado ha construido del amor en la educación, reflejan una interpretación muy cercana a su vivencia personal en el quehacer pedagógico. Así, lo evalúan como una emoción que se siente y se produce como consecuencia de los logros que se consiguen por medio de la labor docente, como se puede apreciar en la siguiente cita: "he sentido amor cuando los alumnos se ven felices egresando de cuarto medio junto con sus familias llorando de felicidad por el logro que su hijo obtuvo" (E1). Este profesor afirma que el amor es una emoción que se encuentra presente en el proceso educativo y que cuando se le siente, le produce una sensación agradable de bienestar, favoreciendo su desempeño profesional expresado de la siguiente forma: "cuando está presente el amor, es andar iluminado, feliz por la vida, en donde los problemas 
que se presentan son un elemento secundario en la vida, encontrándole solución a todo" (E1). Con base en estos relatos, surge el código sentimiento de bienestar subjetivo, representando un estado que es percibido como agradable y deseable, vinculado a un ambiente propicio para el aprendizaje, enunciado así: "uno se da cuenta que hay amor, cuando hay armonía, paz, bienestar y se respira tranquilidad" (E3).

El segundo código "sentimiento transmisible", plantea que el amor se puede transmitir a las demás personas, por ende también se puede recibir, a través del lenguaje verbal y no verbal, de modo que expresa afecto positivo para el bienestar y empatía con la otra persona. Dos expresiones literales de lo anterior lo describen así: "el amor se manifiesta con la empatía hacia las personas y a todo ser viviente, el amor está presente cuando se entrega tiempo y cosas que uno atesora sin condición, sin esperar nada a cambio” (E1) y "el amor es un sentimiento que implica desarrollar hacia distintos seres, una intencionalidad positiva, un querer entregar elementos que promuevan su bienestar" (E2).

El tercer código, construcción en la interacción social, significa que el amor en todos los casos es diferente, puesto que se encuentra compuesto por el aspecto individual (lo que hace única a cada persona) en constante relación con el medio. Hay múltiples factores que influirían en una posible relación construida con amor, con la existencia de la posibilidad de que no se pueda concretar ese vínculo afectivo principalmente por una comunicación carente de valores sociales, como el respeto. Lo anterior se expresa en la consideración del docente de esta forma: "he tenido experiencias que generan reacciones contrarias a una relación amorosa, ya que los alumnos, no tienen idea de qué hay detrás de una clase, no hay respeto, los valores están desvirtuados" (E6). El entorno es parte de la significación que ha emergido del discurso docente, ya que se reconoce que como seres que co-habitamos en un mundo social, tanto docente como alumnado son influenciados por factores externos, como la cultura o experiencias familiares. Por consiguiente, la forma de vivir y significar el amor no está exenta de ser un constructo social y su estado depende de la asociación con diferentes componentes que unidos forman lo humano, lo cual es expresado de la siguiente forma: "el vínculo amoroso se construye, y el ambiente influye mucho, la subjetividad de todos los niños es especial y hay que abordarla como tal, no se pueden estandarizar las relaciones humanas" (E4).

El ambiente educativo es sensible a lo que siente cada participante del proceso y en él interactúan las expresiones de ese sentir de parte del alumnado-profesorado, produciéndose una transformación social, puesto que ninguna sesión de aprendizaje es igual a otra. Entonces, cada vez que el alumnado asiste a su centro educativo, al momento de volver a su hogar ya no es el mismo, debido a que ha sido afectado por sus acontecimientos. La empatía sería una de las manifestaciones que transforma el ambiente y las relaciones educativas, promoviendo la aceptación y el bienestar social

\subsection{Rol del amor en la educación escolar}

La primera función que ha sido atribuida al amor en el proceso educativo, es la vocacional, entendida como el motor afectivo que impulsa al profesorado a realizar una labor comprometida con su alumnado, generándole a la vez, un estado de satisfacción y bienestar, que le retribuye su esfuerzo laboral. En palabras literales, es expresado de esta forma: "para mí el amor es primero que todo, primero debes amar tu profesión, así cada mañana te levantarás con energía, alegría, disposición, amor para enfrentar el día en el aula. Si tú eres feliz, honesto, justo, respetuoso, calmo, amoroso, es lo primero que tus niños van a percibir a través de sus sentidos, te imitarán" (E4). Este componente que nutre la vocación ayudaría a los docentes a ver más allá del currículum escolar y sus exigencias por alcanzar altos índices de calidad nacional o internacional, puesto que también hay otros aspectos que demandan la atención del docente. Entre ellos estaría lo que siente el alumnado en la escuela y sus problemas personales, los cuales son imprevistos e invisibles para las estadísticas del rendimiento educativo. Así expresa un docente esta idea: "un vínculo amoroso es totalmente subjetivo, ya que está compuesto de las sensaciones que provoca la interacción con el otro, por ejemplo, la preocupación de 
ver a un niño que esta con su carita triste, lo cual escapa de las labores planificadas y normativas del currículum del docente" (E5).

La segunda función educativa es favorecer el aprendizaje, por medio de la buena calidad en la interacción social, que sería estimulada con una actitud amorosa, la cual también afecta el entorno y el bienestar subjetivo, tanto del alumnado como del docente. A continuación se presenta una cita que se refiere a lo integrada que se encuentra la dimensión afectiva/emocional con la motivación, en el ciclo de enseñanza-aprendizaje: "a medida que generemos un ambiente amoroso, donde los niños se sientan estimados, respetados y valorados, en donde el profesor se detenga a ayudarlos, se va a crear un vínculo, en donde el alumno con esfuerzo va a presentar una disposición a aprender los contenido más duros del currículum” (E6). Es importante señalar, que el proceso de aprender no debe subestimarse con reglas que desconozcan el contexto, ya que cada situación educativa es única, variando los aspectos que deben ser desarrollados para que se produzca un cierto tipo de aprendizaje. Es evidente que el efecto de los sentimientos transmitidos de los docentes a sus aprendices, no siempre será visible o reconocido por el alumnado, como tampoco en sus aprendizajes, pero si generará por medio de vivencia subjetiva, un modelo positivo de convivir. Por medio de esta expresión, una profesora explica la complejidad de este proceso, donde también se producen emociones de malestar subjetivo en el profesorado: "el adulto si mantiene su actitud amorosa, puede generar cambios y aprendizajes en los alumnos, pero también hay ocasiones en que no hay cambios en ellos, a pesar de que como profesora me esfuerce por mejorar la relación, lo cual es frustrante" (E2).

El tercer código, trata de la ausencia de compromiso con esta dimensión, nombrándose rol irrelevante para la institucionalidad, en donde los participantes del estudio describen que la importancia del amor en la educación se utiliza en ocasiones como una bonita retórica institucional. No obstante, en la práctica no se ve reflejada o vinculada en el quehacer docente. Si el profesorado es más o menos amoroso con el alumnado es un tema que no se evalúa, ni se discute a nivel formal. Para su mejor comprensión, se presenta una cita que expone esta situación: "de acuerdo a la información que emana del Ministerio de Educación, no veo que el énfasis esté en darle importancia a este tema, ya que la educación la enfocan desde una perspectiva cuantitativa, muchas mediciones, planificaciones, para dar con indicadores de logro, reduciendo todo a números, olvidándose de la formación integral del niño" (E3). En un relato similar, un docente señala que percibe un discurso educativo de las autoridades escolares que se encuentra distante de la educación con amor: "el sistema no está dejando ser auténtico, no permite expresarse como uno quiere ser, no propicia el amor, al contrario, propicia el mantener distancia con los niños y los apoderados, evitando involucrarse más allá y eso está fuera de nuestro alcance" (E4).

Educar en los centros escolares tiene que ser un acto significativo para el alumnado escolar, el cual despierte la curiosidad, capte la atención, expanda la creatividad y desarrolle la motivación. Asimismo, se debe desarrollar en un entorno que se promueva el bienestar, por lo que hay que considerar el cerebro emocional, reconociendo al ser humano en su totalidad, como ser que siente y razona al mismo tiempo. Ante esta realidad, el amor se presenta como un componente que estimularía el proceso educativo.

\section{Discusión}

La interpretación del amor que ha sido expresada por el profesorado, coincide en parte con el planteamiento filosófico que lo asocia a las emociones positivas para el bienestar subjetivo (Scheler, 2005). En concreto, es posible sentir el amor, aunque en las apreciaciones del profesorado exista una tendencia a reducirlo a un sentimiento subjetivo. Asimismo, lo reconocen como un sentir que encamina a las personas hacia la felicidad. Esto coincide con los planteamientos de Varela (2009), quien manifiesta que el amor es fundamental desde el nacimiento en la relación materno-infantil, de modo que si logra estar presente será beneficioso en el transcurso de la vida. 
El profesorado también manifiesta que es necesaria la relación entre el amor y la educación, la cual se considera como un proceso de transformación personal. De esta forma, el amor puede lograr un crecimiento en "saber y gobierno", hacia la conquista de la propia autonomía, proceso que se vuelve recíproco, puesto que amar y educar aunque sea en las dosis más leves, transforma al amado y al amante, al educando y al educador (Bazdresch, 2012). Esta transformación, además, puede ser aprovechada por los educadores para mejorar el proceso enseñanza-aprendizaje, pero para ello se requiere de un cambio en la perspectiva curricular pedagógica (Maturana, 2001; Mujica, 2018). Una modificación donde la afectividad reciba un reconocimiento institucionalmente relevante para el proceso pedagógico, como ha sido mencionado por el profesorado. Este posicionamiento pedagógico requiere ser asumido como una meta formal, cuyo como principal propósito sea potenciar un clima propicio de aprendizaje, entendiendo que la emoción es importante tanto para el que aprende como para el que enseña (Freire, 2004). En el mismo sentido, si se desea avanzar en este tema hay que conectar los significados emocionales con los aprendizajes, ya que "nada se puede conocer más que aquello que se ama, aquello que nos dice algo" (Mora, 2017, p. 71). Entonces, el significado afectivo que construye el alumnado en los procesos educativos repercutirá en su motivación intrínseca, por ello el profesorado que asuma un rol activo en esta dimensión podrá ayudarlos en su aprendizaje. Asimismo lo entienden Guerrero y Cárdenas (2009), precisamente, cuando plantean que "la influencia del profesorado puede ser determinante para el acercamiento del alumnado hacia el conocimiento, hasta tal punto que puede convertirse en la génesis de los procesos motivacionales hacia el aprendizaje" (p. 217).

Uno de los descubrimientos científicos que da sentido a un cambio en la forma de entender la educación en el proceso de escolarización, es el de las neuronas espejos o neuronas de la empatía (Morris, 2014). Este hallazgo fundamenta la importancia de las emociones positivas para el bienestar subjetivo que suscitaría el amor (Mujica, 2019). Así, por medio de aquellas neuronas, el alumnado puede hacer propios los gestos, posturas, expresiones y emociones del docente, sucediendo lo mismo al revés, y así, el docente puede percibir esas expresiones del alumnado (Morris, 2014). La empatía, ha sido reconocida en los relatos otorgados por el profesorado como una expresión de su amor en la educación, señalando que si bien se les exige cumplir el currículo, no dudan en detenerse a atender otros aspectos que involucran el sentir de los discentes. Por lo mismo, declaran que no pueden ser indiferentes a las emociones del estudiantado que les produce un malestar subjetivo, como por ejemplo la tristeza. Al igual que esta interpretación, la empatía es entendida como una capacidad que expresa amor, predisponiendo a la persona no solo a sentir el sufrimiento del otro, sino que también a sentir su dicha y participar de su alegría (Carpena, 2016). En consecuencia, una persona que logra amar por medio de la educación se relacionaría con una buena capacidad empática, alcanzandola consecución de las metas altruistas con mayor facilidad, las cuales requieren dedicar grandes esfuerzos para el bienestar de los demás (Mujica, 2019).

El amor también fue vinculado a la vocación profesional y se destacó en el relato docente, que se encuentra presente al momento de desempeñar las funciones pedagógicas, lo cual es de gran ayuda para la motivación y el bienestar personal. En consecuencia, también el alumnado que se encuentra bajo su responsabilidad se beneficiaría de la vocación docente, quienes podrán construir interacciones sociales fundadas en la aceptación y la comprensión emocional (Bisquerra, 2005). Esta representación tiene relación con algunos estudios sobre la vocación docente en los que participaron estudiantes y docentes en ejercicio de Pedagogía Educación Física, quienes representaron la vocación como una construcción subjetiva que emerge desde sus experiencias afectivas significativas (Mujica y Orellana, 2016; Mujica y Orellana, 2018; Mujica y Orellana, 2019). En el mismo sentido se ha señalado que la vocación tendrá un gran amor al ámbito que se dirige (Gracia, 2007, p. 814). En síntesis, una presencia amorosa del cuerpo docente en la educación escolar, requiere amarse a sí mismo, amar al alumnado y amar su labor (Toro, 2013; Toro, 2017).

El código que describe la irrelevancia del amor en la praxis educativa, expresa el problema de deshumanización presente en las finalidades educativas, marcado por la presión que tienen los docentes de producir buenos resultados en las pruebas estandarizadas (Flórez y Oyarzún, 2016). Como una forma de 
enfrentar esta situación que se erige como la principal responsable del agobio laboral en Chile, surgió una campaña social llamada alto al SIMCE (Sistema de Medición de la Calidad Educativa), la cual evidencia el daño social y emocional que produce esta prueba en el profesorado. Daño que principalmente se encontraría asociado a los estímulos o premios por los resultados en dichas evaluaciones estandarizadas, las cuales se pueden transformar en una recompensa económica o en un castigo social (Campos-Martínez y Guerrero, 2016). Por consiguiente, la predominante concepción tecnocrática junto a las políticas neoliberales en la educación chilena, caracterizadas por la privatización y el desmantelamiento de la educación pública, han conducido a una grave deshumanización de la educación. Dicha situación, se ve reflejada en los altos índices de segregación escolar, falta de reconocimiento a la profesión docente y agobio del profesorado (CamposMartínez y Guerrero, 2016; Contreras y Macías, 2002; García-Huidobro, 2007; Mujica y Orellana, 2019; Oliva, 2008; Puga, 2011; Cavieres, 2014). Tal como se ha señalado, el currículo escolar chileno ha sido muy criticado por su carácter reproductivo de los aprendizajes y su perspectiva tecnocrática dominante de estandarización (Donoso, 2005; Moreno, Gamboa y Poblete, 2014; Moreno, Rivera y Trigueros, 2014; Oliva y Gascón, 2016). Por ello, es imprescindible que sucedan cambios profundos en la educación escolar chilena, para así avanzar hacia una educación transformadora, crítica, basada en el diálogo, el amor y la cooperación (Carter-Thuillier y Moreno, 2018; Freire, 2004).

\section{Conclusiones}

De acuerdo con las ideas que se han expuesto a lo largo de este trabajo, se concluye que es posible sentir el amor en la práctica docente, el cual se percibe como un sentimiento asociado a una experiencia de bienestar subjetivo, que en ocasiones responde a los logros del alumnado, como a sus expresiones emocionales, con énfasis en los momentos en los cuales se demuestran felices por observar sus metas cumplidas. También el profesorado reconoce que el amor se puede transmitir a las demás personas y, a su vez, pueden percibir cuando se les expresa, por lo que el proceso educativo puede realizarse con la presencia de este fundamental componente afectivo-moral. De esta forma, se estaría promoviendo un adecuado ambiente de aprendizaje, compuesto por relaciones sociales fraternas, agradables, acogedoras y empáticas, donde su sentir personal posee un espacio relevante.

Si bien se ha generado una aproximación hacia los significados sobre el amor en el ámbito educativo creados por profesionales que se desempeñan en educación pública, es necesario que se desarrollen otros estudios con diferentes grupos del profesorado, del equipo directivo, del alumnado y de las familias. Esto permitirá construir una teoría del amor que analice el pensar y el sentir de una gran parte de los protagonistas de la praxis educativa.

La principal transferencia que se propone para este trabajo, es la de generar conciencia en el profesorado con respecto al rol que posee y que debería tener el amor en el proceso educativo. Este es el primer paso para comenzar a abrir espacios formales a conceptos como la empatía, las emociones o el bienestar, que si bien se pueden encontrar declarados en algunos documentos oficiales del MINEDUC, en el quehacer docente suelen estar muy ausentes. Trabajar por humanizar en la actualidad dentro de los procesos educativos, que se han visto muy afectados por las políticas neoliberales, provocando que los factores cualitativos sean desvalorizados, es una responsabilidad política de toda la sociedad. Por lo tanto, transformar dicha realidad es un desafío del cual la totalidad de la responsabilidad no puede implicar solo al magisterio, aunque sin duda, es el gremio docente que debe liderar estas importantes reformas educativas.

\section{REFERENCIAS}

Araque, N. (2010). La educación en el amor. Revista Crítica, 966, 60-65. 
Mag. Felipe Mujica Johnson, et al. Apreciaciones del profesorado chileno sobre una educación basad...

Anguera, M., Arnau, J., Ato, M., Martínez, R., Pascual, J. y Vallejo, G. (1998). Métodos de investigación social. Madrid, España: Síntesis Psicológica.

Barbera, N. y Inciarte, A. (2012). Fenomenología y hermenéutica: dos perspectivas para estudiar las ciencias sociales y humanas. Revista Multiciencias, 12(2),199-205.

Bazdresch, M. (2012). La conversación educativa: un acto amoroso. Revista Interuniversitaria de Formación del Profesorado, 26(2), 75-88.

Bisquerra, R. y Pérez, N. (2007). Las competencias emocionales. Educación XXI, 10(1), 61-82.

Bisquerra, R. (2005). La educación emocional en la formación del profesorado. Revista Interuniversitaria de Formación del Profesorado, 54, 95-114.

Bogdan, R. y Taylor, S. (2009). Introducción a los métodos cualitativos de investigación. Barcelona, España: Paidós.

Cabezas, M. (2013). Juicios morales y fronteras biológicas: más allá de la frontera razón emoción. Revista Arbor, 189(762), 1-1.

Campos-Martínez, J. y Guerrero, P. (2016). Efectos indeseados de la medición de la calidad educativa en Chile. La respuesta de la sociedad civil. Cadernos Cedes, 36(100), 355-374.

Carpena, A. (2016). La empatía es posible. Educación emocional para una sociedad empática. Bilbao, España: Desclée de Brouwer.

Carter-Thuillier, B. y Moreno, A. (2018). Globalización económica, postmodernidad y sistema educativo: contradicciones y alternativas desde una Educación Física crítica. Estudios Pedagógicos, 43(3), 103-117.

Cavieres, E. (2014). La calidad de la educación como parte del problema. Educación escolar y desigualdad en Chile. Revista Brasileira de Educaçao, 19(59), 1033-1051.

Chalita, G. (2006). Pedagogía del amor: las historias universales y los valores de las nuevas generaciones. Madrid, España: Pensar, publicar, creer.

Contreras, D. y Macías, V. (2002). Desigualdad educacional en Chile: Geografía y Dependencia. Cuadernos de economia, 39(118), 395-421.

Descartes, R. (2011). Meditaciones metafísicas. Madrid, España: Alianza.

Donoso, S. (2005). Reforma y política educacional en Chile 1990-2004: Neoliberalismo en crisis. Estudios Pedagógicos, 31(1), 113-135.

Ferrés, J. y Masanet, M. (2017). La eficacia comunicativa en la educación: potenciando las emociones y el relato. Comunicar, 25(52), 51-60.

Flórez, M. y Oyarzún, G. (2016). Resultados SIMCE y Plan de Evaluaciones 2016-2020: Nudos críticos y perspectivas de cambio. Cuaderno de Educación, 73, 1-11

Freire, P. (2004). Cartas a quien pretende enseñar. Buenos Aires: Siglo XXI.

Fromm, E. (1959). El arte de amar. Barcelona, España: Paidós.

García-Fariña, A., Jiménez, F. y Anguera, M. (2016). Análisis observacional del discurso docente del profesorado de educación física en formación a través de patrones comunicativos. Cuadernos de Psicología del Deporte, 16(1), 171-182.

García-Huidobro, J. (2007). Desigualdad educativa y segmentación del sistema escolar. Consideraciones a partir del caso Chileno. Revista de Pensamiento Educativo, 40(1), 65-85.

Gracia, D. (2007). La vocación docente. Anuario Jurídico y Económico Escurialense, 40(1), 807-816.

Guerrero, A. y Cárdenas, A. (2009). Influencia de la competencia emocional docente en la formación de procesos motivacionales e identitarios en estudiantes de Educación Secundaria. Una aproximación desde la memoria autobiográfica del alumnado. Revista de Investigación Educativa, 27(1), 203-222.

Hernández, V. (2017). Las competencias emocionales del docente y su desempeño profesional. Alternativas en psicología, 37, 79-92.

Jodelet, D. (2011). Aportes del enfoque de las representaciones sociales al campo de la educación. Espacios en blanco. Revista de Educación, 21(1), 133-154. 
Jordan, J. y Méndez, J. (2017). Rasgos esenciales de los profesores excelentes en su relación con los alumnos tras el visionado de películas pedagógicamente valiosas. Estudios sobre educación, 33(1), 103-126.

Maturana, H. (2001). Emociones y lenguaje en educación y politica. Santiago de Chile: Dolmen.

Ministerio de Educación. (2009). Formación en afectividad y sexualidad. Santiago, Chile: Gobierno de chile.

Ministerio de Educación. (2010). Ley general de Educación. Santiago, Chile: Biblioteca del Congreso Nacional.

Ministerio de Educación. (2013). Formación en sexualidad, afectividad y género. Santiago: Gobierno de Chile.

Mora, F. (2017). Neuroeducación. Solo se puede aprender aquello que se ama. Madrid, España: Alianza.

Moreno, A., Rivera, A. y Trigueros, C. (2014). Sistema de medición de la calidad de la Educación Física Chilena: un análisis crítico. Movimento, 20(1), 145- 167.

Moreno, A., Gamboa, R. y Poblete, C. (2014). La educación física en chile: análisis crítico de la documentación ministerial. Revista Brasileira de Ciências do Esporte, 36(2), 411-427.

Moreno, A. y Poblete, C. (2015). La educación física chilena y su profesorado: proponiendo algunos retos para la investigación en el área. Retos: nuevas tendencias en educación física y deporte y recreación, 28, 291-296.

Moreno, G. (2000). Introducción a la metodología de la investigación educativa II. México: Progreso.

Morris, M. (2014). La neuroeducación en el aula: neuronas espejo y la empatía docente. Revista la vida y la historia, 3(2), 9-18.

Mujica, F. (2018). Educar y suscitar emociones en la educación: Análisis crítico de su contribución al desarrollo moral. Revista de la Facultad de Educación de Albacete, 33(2), 15-27.

Mujica, F. (2019). Suscitar buenas emociones en la educación formal: Análisis según la ética de Max Scheler. Revista Dilemas Contemporáneos: Educación, Política y Valores, 6(3), 1-15.

Mujica, F. y Orellana, N. (2019). Deseos vocacionales que incentivan a la formación docente en Educación Física. Revista Educación, 43(1), 1-13. doi: https://doi.org/10.15517/revedu.v43i1.30013

Oliva, M. (2008). Política educativa y profundización de la desigualdad en Chile. Estudios Pedagógicos, 34(2), 207-226.

Oliva, M. y Gascón, F. (2016). Estandarización y racionalidad política neoliberal: bases curriculares de Chile. Cadernos Cedes, 36(100), 301-318.

Ortiz, A. (2009). Educación infantil: afectividad, amor y felicidad, curriculo, lúdica, evaluación y problemas de aprendizaje. Barranquilla: Litoral.

Pascal. (1967). Pensamientos. Madrid, España: Espasa-Calpe.

Pérez-López, R., Morales-Sánchez, V., Anguera, M. y Hernández-Mendo, A. (2016). Modelo tridimensional de la calidad en organizaciones deportivas: calidad emocional em usuários/as infantiles. Cuadernos de Psicología del Deporte, 16(1), 143-150.

Puga, I. (2011). Escuela y estratificación social en Chile: ¿Cuál es el rol de la municipalización y la educación particular subvencionada en la reproducción de la desigualdad social? Estudios Pedagógicos, 37(2), 213- 232.

Scheler, M. (2005). Esencia y formas de la simpatía. Salamanca, España: Sígueme.

Stake, R. (1995). The art of case study research. London, Reino Unido: Sage.

Stake, R. (2007). Investigación con estudio de casos. Madrid, España: Morata.

Rapley, T. (2014). Los análisis de la conversación, del discurso y de documentos en investigación cualitativa. Madrid, España: Morata.

Rojas, E. (1993). Una teoría de la felicidad. Madrid, España: Dossat.

Toro, J. (2013). ¿Metodologías de la interioridad? La presencia del maestro. En L. López (Ed.), Maestros del corazón. Hacia una pedagogia de la interioridad (pp. 295-237). Madrid, España: Wolters Kluwer.

Toro, J. (2017). Educar con “co-razón”. Bilbao, España: Desclée de Brouwer.

Varela, P. (2009). El amor. En E. Fernández-Abascal (Ed.), Emociones positivas (pp.149-166). Madrid, España: Pirámide. 
Mag. Felipe Mujica Johnson, et al. Apreciaciones del profesorado chileno sobre una educación basad...

Vargas-Jiménez, I. (2012). La entrevista en la investigación cualitativa: nuevas tendencias y retos. Revista Calidad en la Educación Superior, 3(1), 119- 139.

Vergara, M. (2008). La naturaleza de las representaciones sociales. Revista latinoamericana de ciencias sociales, niñez y juventud, 6(1), 55-80.

Zaccagnini, J. (2008). La comprensión de la emoción. Una perspectiva psicológica. En M. Jiménez (Ed.), Educación Emocional y Convivencia en el Aula (pp. 31-84). Madrid, España: Ministerio de Educación, Política Social y Deporte.

Todos los derechos reservados. Universidad de Costa Rica. Esta revista se encuentra licenciada con Creative Commons. Reconocimiento-NoComercial-SinObraDerivada 3.0 Costa Rica. Correo electrónico: humanidades@ucr.ac.cr/Sitio web: http: //revistas.ucr.ac.cr/index.php/humanidades

CC BY-NC-ND 